\title{
Corela
}

Cognition, représentation, langage

3-1 | 2005

Vol. $3, n^{\circ} 1$

\section{Problématique des grands groupes et didactique du français au Cameroun}

David Ngamassu

\section{OpenEdition}

\section{Journals}

Édition électronique

URL : http://journals.openedition.org/corela/503

DOI : $10.4000 /$ corela.503

ISSN : $1638-573 \mathrm{X}$

\section{Éditeur}

Cercle linguistique du Centre et de I'Ouest - CerLICO

Référence électronique

David Ngamassu, «Problématique des grands groupes et didactique du français au Cameroun », Corela [En ligne], 3-1 | 2005, mis en ligne le 04 juillet 2005, consulté le 30 avril 2019. URL : http:// journals.openedition.org/corela/503; DOI : 10.4000/corela.503

Ce document a été généré automatiquement le 30 avril 2019

\section{(c) (i) (2)(2)}

Corela - cognition, représentation, langage est mis à disposition selon les termes de la licence Creative Commons Attribution - Pas d'Utilisation Commerciale - Partage dans les Mêmes Conditions 4.0 International. 


\title{
Problématique des grands groupes et didactique du français au Cameroun
}

\author{
David Ngamassu
}

\section{Prolégomènes}

1 La Déclaration finale des travaux de la $45^{\mathrm{e}}$ session de la CONFEMEN ${ }^{2}$ tenue à Yaoundé en 1994, après avoir dressé un tableau sombre et alarmant de la situation de l'école en Afrique noire francophone : programmes scolaires inadaptés, savoirs décontextualisés et mal maîtrisés, sous-scolarisation, mal-scolarisation, déscolarisation, mauvaises politiques scolaire, etc., recommande formellement une réorientation de ses objectifs et finalités, si l'on veut que dans les décennies à venir, elle continue à remplir sa mission de formation de la jeunesse, et partant de diffusion de la langue française. Plus d'une décennie s'est écoulée depuis ces assises, et l'on peut constater que la situation de l'école et de l'enseignement du français en Afrique et donc au Cameroun n'a pas évolué de manière positive.

2 La présente étude, qui est une réflexion critique sur la situation de l'enseignement du français au Cameroun soulève quelques-uns des problèmes majeurs qu'il pose en ce début du XXI ${ }^{e}$ siècle : à quels types de problèmes spécifiques est-il confronté ? Face à son acclimatement en Afrique noire et partant, à l'émergence des normes endogènes, l'institution scolaire classique pourra-t-elle continuer à assurer son rôle de vecteur exclusif de sa diffusion et de gardienne de la norme linguistique ? A l'heure de la mondialisation linguistique, quelles politiques scolaires des langues mettre en place au Cameroun, afin d'assurer aux générations futures un enseignement efficace du français et une amélioration durable de la qualité de cet enseignement? Quelle stratégie adopter pour permettre à l'école moderne d'offrir à une population scolaire en constante croissance un enseignement $d u$ français de qualité ? Telles sont quelques-unes des interrogations que soulève la présente étude, dont le but n'est pas d'épuiser le questionnement méthodologique en didactique des langues, mais de réfléchir sur les 
voies et moyens d'une refondation de l'école et donc, de l'enseignement du français au Cameroun en particulier. La didactique du français est un champ pluridisciplinaire, tellement vaste qu'on ne saurait avoir l'ambition d'en explorer tous les aspects dans cette étude ; nous limiterons donc volontairement à l'un des problèmes majeurs auxquels elle est confrontée au Cameroun depuis plusieurs décennies : les classes à effectifs pléthoriques et les problèmes que pose sa gestion quotidienne. L'examen de toute réalité pluridimensionnelle implique deux démarches scientifiques possibles : l'une, analytique, consiste à en distinguer, séparément, les différents éléments, et à les considérer comme des entités autonomes; l'autre, holistique, cherche à établir des liens de causalité entre les différents éléments de l'ensemble. La deuxième démarche, encore appelée approche systémique, analyse toute situation comme un système constitué d'éléments différents en interaction réciproque les uns sur les autres, et dont l'interdépendance assure la cohésion et l'unité de l'ensemble. En effet, la réussite ou l'échec de l'enseignement du français au Cameroun dépend de la conjonction de plusieurs facteurs inter-reliés et d'une multitude d'intentions, de pratiques et d'attitudes. La recherche de solutions aux problèmes de la didactique du français au Cameroun ne saurait donc s'appuyer sur un modèle de causalité unidirectionnelle, c'est pourquoi l'approche systémique, démarche à la fois descriptive et prospectiviste, semble à l'heure actuelle l'une des voies les plus fécondes pour une analysediachronique et synchronique de l'école au Cameroun.

3 Parmi les problèmes auxquels elle est confrontée au Cameroun, figure la croissance démographique exponentielle, dont le corollaire est l'augmentation constante des demandes en matière d'éducation. En effet, depuis trois décennies, la croissance des populations scolarisables constitue un casse-tête pour les responsables éducatifs camerounais, qui font face à des difficultés de plus en plus insurmontables. Les salles de classe prévues pour accueillir une trentaine d'élèves il y a vingt ans en accueillent aujourd'hui deux, voire trois fois plus. Quelques chiffres illustrent mieux la situation : en 1961 la population scolaire au niveau primaire était de 421000 élèves, en 1968-69 elle est passée à 938000 élèves, puis à 2400000 en 90-91; en 2003-2004, elle se situait à 3500000 élèves. Dans le même temps le nombre de maîtres est passé de 13407 en 1970 à 38429 en 1990, et se situe en 2004-2005 à moins de 50 000, dont plus des deux tiers constitués de maîtres vacataires. Quant à celui des salles de classe, il n'a pas suivi cette croissance. Déjà en 65-66, sur les 4954 salles que comptait l'enseignement primaire officiel, 390 avaient plus de 70 élèves, et 761 comptaient plus de 80 élèves. Raymond Lallez constate en 1974 que "c'est au niveau de la Section d'initiation que les effectifs d'élèves par classe dépassent souvent la centaine ${ }^{13}$. La première conséquence de cette surpopulation scolaire est évidemment le faible rendement interne de l'institution scolaire. En effet d'après une étude sérieuse, publié en 1973, 43\% des élèves abandonnaient l'école à la fin de la quatrième année, tandis que $31 \%$ redoublaient eu que seulement $15 \%$ passaient le certificat de fin d'études primaires élémentaires ${ }^{4}$. Dix ans plus tard, Engilbert. Mveng constate qu'en 1985 que "sur mille enfants entrés au Cours d'initiation, un seul arrive au baccalauréat ${ }^{\prime 1}$.Une autre décennie après une étude réalisée par Henri Tourneux et Olivier Iyebi-Mandjeck à Maroua (Province de l'Extrême-Nord) révèle que seulement $42 \%$ des élèves qui sont entrés à la Section d'Initiation en 92-93 sont arrivés au Cours moyen deuxième année, et moins de $14 \%$ ont obtenu le Certificat d'études primaires. D'après cette étude, "86\% des élèves entrés à la SIL en 1992 n'auront jamais passé leur certificat d'études primaires, 90\% n'entreront jamais en secondaire ${ }^{15}$. Trente ans après l'indépendance, ni le nombre d'élèves par classe, ni les rendements internes et externes de l'école n'ont connu une amélioration qualitative subséquente. Aline Cook note qu'au Cameroun le ratio élèves-professeurs est loin de 
l'idéal, car en 1990 "la norme officielle prévoit 40 élèves par professeur. Or on voit parfois jusqu'à 200 élèves pour un seul professeur". Ces chiffres illustrent bien l'une des tristes réalités auxquelles est confrontée l'école, et partant l'enseignement du français au Cameroun : les grands groupes ou classes à effectifs élevés. Alors que dans la plupart des pays industrialisés, où les taux de natalité sont en constante décélération depuis plusieurs années, la tendance est à une réduction de la taille des groupes-classes, au Cameroun, comme dans la plupart des pays africains, elle connaît une croissance vertigineuse ; d'où la problématique des classes à effectifs pléthoriques, qui couplée aux autres problèmes épineux : insuffisance de ressources humaines et financières, inadaptations et inadéquation des programmes et des outils pédagogiques, inertie du système scolaire engluée dans la tradition, et réticente, voire réfractaire à toute transformation radicale rendent l'école inefficace et inapte à la diffusion du français.

\section{Genèse de la pédagogie des grands groupes}

La problématique de la pédagogie des grands groupes a été posée pour la première fois en mars 1984, lors de la réunion à Khartoum de l'APFA, (Association des Professeurs de Français en Afrique). En juin 1985, en marge des journées 'Pratique du dialogue des cultures pour les échanges éducatifs' organisées au CIEP (Centre International d'Etudes Pédagogiques) de Sèvres par la FIPF (Fédération Internationale des Professeurs de Français), les professeurs de français en Afrique noire francophone décident de se réunir en atelier spécialisé, pour jeter les bases de la réflexion sur l'enseignement du français dans les classes à effectifs pléthoriques, phénomène très répandu dans la plupart des grandes villes africaines. Il se dégage des travaux de cet atelier que, compte tenu de la croissance démographique incontrôlée que connaissent les pays africains, du déficit chronique en mobilier scolaire, du manque d'outils didactiques, et de l'insuffisance quantitative et qualitative du personnel enseignant et des moyens financiers, le problème des classes surchargées n'est pas une situation conjoncturelle. S'appuyant sur les diagnostics dressés par plusieurs experts, les participants à cet atelier sont donc unanimes que pour longtemps encore, les enseignants de français en Afrique noire sont condamnés à travailler dans des classes surchargées. Par conséquent, face à l'improbabilité du retour à une situation de classes à effectifs raisonnables, il faut faire contre mauvaise fortune bon cœur, en mettant en place des démarches méthodologiques et didactiques adaptées à ce contexte particulier.

5 Depuis l'appel de Khartoum, plusieurs rencontres consacrées partiellement, ou entièrement à la pédagogie des grands groupes ont été organisées en France ou dans certains pays africains : à Djibouti en novembre 1985, Colloque du Sèvres en juin 1986, Séminaire de Brazzaville en novembre 1986, rencontres de l'Ile Maurice en février 1987, Séminaire de Sèvres en février 1988, Séminaire de Thiès (Sénégal) en novembre-décembre 1988, réunions de Bordeaux en janvier 1989 et décembre 1990, séminaire de N'Djamena en 1991, journées de Dakar en juillet 1995, etc. La fréquence et surtout le nombre de journées, rencontres et séminaires consacrés à la réflexion sur la pédagogie des grands groupes témoignent de l'importance grandissante accordée à cette question. La publication du Répertoire méthodologique sur les techniques d'organisation et d'enseignement dans les classes à effectifs pléthoriques par la CONFEMEN, (Conférence des Ministres de l'éducation des pays ayant en commun le français), en juillet 1991, marque un tournent décisif dans la réflexion sur la pédagogie des grands groupes. 


\subsection{Définition de la pédagogie des grands groupes}

6 Tout le monde s'accorde sur le fait qu'au-delà d'un certain seuil, l'action de l'enseignant en classe n'est plus efficace, dans la mesure où il n'y a plus d'interaction possible au sein du groupe-classe. Cependant il est difficile de fixer avec précision ce seuil; par conséquent on ne peut pas définir avec exactitude ce qu'est un grand groupe. Une définition consensuelle fixe à quarante-cinq, ou au plus à cinquante, la moyenne au-delà de laquelle on est dans une situation de classe à effectifs élevés. Il faut dire cependant qu'il ne s'agit que d'un seuil relatif, car au Cameroun rares sont les classes, même en zones rurales, ayant moins de cinquante élèves. Dans les grandes villes, la moyenne se situe généralement entre cent et cent-cinquante, avec des pics à plus de deux cents élèves dans certains quartiers populeux. Si l'on s'en tient uniquement aux expressions employées pour désigner les grands groupes : 'classes pléthoriques', 'classes surchargées', 'classes à effectifs élevés', on peut dire que le nombre d'élèves constitue le principal critère définitionnel retenu. Pourtant d'autres éléments doivent être pris en compte, notamment les moyens matériels, la formation des enseignants, la motivation des élèves, l'adéquation des outils méthodologiques, etc.

7 Face à cette inadéquation, seules des solutions propres à chaque contexte particulier constituent la voie de survie pour l'école au Cameroun. Les demandes en salles de classe et en enseignants croissent de façon exponentielle, et malgré tous les efforts déployés, le pays n'est plus en mesure d'offrir un enseignement de qualité. Le système de double flux, proposé dans les années soixante-dix, et qui était censé résoudre le problème d'effectifs en optimisant l'exploitation des salles de classe, a permis de le résorber provisoirement, en multipliant par deux les capacités d'accueil des salles de classe. Pratiqué surtout dans les grandes villes, ce système appelé 'classes à mi-temps' consiste à atténuer la pénurie de locaux, en en allouant une même salle à deux classes distinctes, qui l'utilisent en demijournée de manière alternée : les élèves de la classe A sont scolarisés une semaine sur deux entre sept heures et douze heures, tandis que ceux de la classe B sont scolarisés les après-midi, de douze heures trente minutes à dix-sept heures.

Ce système a pour inconvénient majeur de réduire considérablement le nombre d'heures de cours effectifs par semaine. Au Cameroun l'année scolaire, déjà réduite à trente et une semaines (à cause des vacances de fin d'année particulièrement longues), contre trentesix semaines fixées par l'UNESCO, est fortement grevée par les multiples jours fériés et d'interminables fêtes légales et 'illégales' : ( $1^{\mathrm{er}}$ janvier, fête de la jeunesse, du travail, fête nationale, fête du Mouton, fête du Ramadan, fête de l'Ascension, fête de la Réunification, sans compter les multiples ponts et autres jours fériés décrétés ou non, à l'occasion des tournées en provinces des responsables politiques et administratifs à divers niveaux). Malgré la généralisation du système de double flux dans les grandes villes, et de plus en plus dans les villes moyennes et les villages, au Cameroun, l'école primaire offre généralement le spectacle désolant des classes où plusieurs dizaines d'élèves, assis quelquefois à même le sol, s'entassent les uns sur les autres, dans des salles exiguës mal aérées et sans éclairage, encadrés par des enseignants qui, parce que pas préparés à la gestion de ces situations de crise, croulent sous le poids des tâches diverses, en dépit de leur dévouement. En réalité dans les grandes villes camerounaises le véritable problème est plus le manque de salles de classe, que l'insuffisance quantitative d'enseignants. L'expérience montre que dans les villes de Douala et Yaoundé par exemple, il y 
généralement deux à trois fois plus de maîtres dans les écoles que de salles de classe disponibles. Dans la plupart des classes en milieu urbain, on trouve deux voir trois maîtres dans une même classe, alors que dans le même temps dans certaines écoles de campagne, un seul maître est obligé d'enseigner deux voire trois classes. Dans ces classes tenues par deux voire trois maîtresses la pratique courante consiste généralement à se partager quotidiennement les tâches : par exemple l'une s'occupe de la discipline, pendant que l'autre enseigne et que la troisième corrige les cahiers des élèves.

\subsection{Le grand groupe : une fatalité ?}

On peut se demander cependant si la situation de grand groupe est une fatalité, c'est-àdire une situation contre laquelle on ne peut rien, et qu'il faut assumer. En se basant sur les données disponibles et les prospectives, on est tenté de répondre par l'affirmative. Car même les études les plus optimistes n'entrevoient pas, dans l'immédiat, ou même dans un avenir proche, de solutions adéquates. Le taux de natalité demeure très élevé au Cameroun, si on le compare aux courbes de croissance économique et du développement infrastructurel. Nous pensons cependant que faire l'apologie de la pédagogie des grands groupes, c'est remettre en cause les fondements mêmes de l'éducation, dans la mesure où chaque enfant qui arrive à l'école, comme chaque malade qui va voir le médecin, est un cas unique. On ne saurait donc imaginer une situation scolaire de thérapie de groupe. Même dans les situations critiques de grande pandémie, aucun médecin n'envisagerait de regrouper ses malades et de les soumettre à un traitement collectif ou à une thérapie collective. Ilne lui arriverait pas non plusl'idée de demander aux malades moins souffrants de s'occuper des plus malades.

En effet, en milieu scolaire camerounais, la pratique assez répandue consistant à demander à certains élèves plus âgés de s'occuper d'autres élèves ressemble fort à une telle situation. Pour certains cette pratique est condamnable, car plus l'enfant est jeune, plus il est fragile et délicat, et par conséquent plus il a besoin d'une approche pédagogique particulière, appropriée à sa situation socio-affective, qui est fondamentalement différente de celle des élèves plus âgés. Choisir, à ce stade fragile de sa croissance, de le confier à d'autres élèves, fussent-ils plus grands ou plus forts, c'est en quelque sorte le sacrifier. Certes le travail en grands groupes contribue au processus de socialisation de l'enfant, mais il ne saurait constituer une démarche pédagogique. Car parler d'une approche pédagogique signifie qu'on est face à une situation stable et acceptable. On ne saurait donc ériger en modèle pédagogique une situation pathogène. En effet, les grands groupes sont une situation de crise, et les remèdes aux situations de crise relèvent du ponctuel. Prétendre que la situation de grands groupes est une condition favorable d'enseignement n'est pas, à notre avis, une position pédagogiquement défendable ; car plus un groupe est grand, plus il est difficilement gérable, et plus l'homme (et à plus forte raison le jeune enfant), y paraît fragile face à l'immensité de la foule. André de Peretti, citant Newcomb, affirme que "lorsque la taille du groupe augmente, les ressources tendent à augmenter" . Mais ceci ne saurait s'appliquer au groupe-classe. Car s'il avait été prouvé que les grands groupes sont susceptibles de donner des résultats meilleurs, il y a longtemps que toutes les salles de classe auraient été transformées en amphithéâtres, ce qui évidemment en réduirait le coût de fonctionnement, tout en augmentant leurs rendements. 
11 Nous ne prétendons pas cependant que les petits groupes aient un rendement absolument meilleur. En effet les exemples des écoles des zones rurales au Cameroun, où les effectifs dépassent rarement une cinquantaine d'élèves par classe, montrent que les résultats ne sont pas nécessairement fonction de la taille du groupe-classe. De même, dans les grandes villes, certaines écoles qui accueillent en majorité les 'enfants des riches', et dont les effectifs par classe ne dépassent jamais 40-50 élèves, (l'Ecole du Centre Administratif et l'Ecole Bilingue de Bastos à Yaoundé par exemple), ne sont pas nécessairement les meilleures en termes de rendement scolaire. Ces remarques faites, il faut cependant se rendre à l'évidence, et reconnaître que si la pédagogie des grands groupes n'est pas la solution idéale, elle constitue néanmoins la seule possible à l'heure actuelle. Par conséquent, faute de pouvoir trouver une solution acceptable, le pragmatisme recommande de reconnaître que la pédagogie des grands groupes, solution provisoirement définitive, ou définitivement provisoire, est la seule applicable au Cameroun aujourd'hui.

\subsection{Une solution provisoirement définitive}

12 Mais on ne saurait cependant se résigner à son triste sort, ni se faire le chantre de l'échec de toute pédagogie en Afrique. Il se pourrait que la loi du grand nombre, qui constitue aujourd'hui, pour les pays africains, un obstacle quasi insurmontable, devienne demain un véritable atout pour leur développement. C'est pourquoi les recherches en pédagogie des grands groupes méritent d'être encouragées et soutenues. Certes la taille du groupeclasse peut favoriser ou compliquer la tâche de l'enseignant, suivant le type d'activités. Mais il est difficile de prouver scientifiquement que plus la classe est surchargée, plus elle est dynamique, comme l'affirment certains chercheurs, s'appuyant sur la conception médiévale de la pédagogie défendue par Jean Amos Comenius qui écrit :

"je soutiens, non seulement qu'un seul maître pourrait diriger une centaine d'élèves, mais aussi que cela convient mieux et est avantageux pour lui et pour les élèves. Le maître remplira sans nul doute ses fonctions avec d'autant plus de plaisir qu'il aura devant lui des élèves plus nombreux. [ ... ] lorsque l'auditoire du maître est peu nombreux, fatalement telle ou telle de ses paroles ne sera pas recueillie par les oreilles de tous, tandis que s'il est nombreux, chacun attrape autant qu'il peut"8

On ne saurait cependant proposer aujourd'hui, comme solution au problème des classes à effectifs élevés, cette approche méthodologique vieille de plusieurs siècles. Si l'idée défendue par Jean Amos Comenius d'ouvrir l'école au plus grand nombre d'enfants possible, donc de favoriser un enseignement de masse est certes conforme à la politique scolaire de la plupart des pays africains, le véritable problème est l'inadéquation entre cette politique de l'école des masses et les moyens humains et matériels disponibles. Ce problème se pose en termes diamétralement opposés, car la question en Afrique est de savoir comment gérer le flux de plus en plus important d'enfants qui aspirent légitimement au savoir. Il convient de dire que tant que ces pays n'auront pas mis en place une véritable politique de limitation des naissances, les grands groupes constitueront toujours un problème fondamental pour l'école ; par conséquent les enseignants africains seront éternellement condamnés à la débrouillardise méthodologique. Car ce qui fait la particularité de la pédagogie des grands groupes, c'est l'esprit d'initiative dont l'enseignant est en permanence obligé de faire montre, dan l'organisation de la classe. Il est condamné à faire avec le peu de moyens dont il dispose, de se débrouiller. Comme le note si bien André de Peretti, 
"chaque dimension de groupe définit des possibilités de travail spécifiques de groupe par rapport à des objectifs spécifiques et différentiels. On ne fait pas la même chose avec un petit groupe, un groupe moyen ou un grand groupe en termes d'objectifs, pas plus qu'en termes d'organisation. Dans chaque cas, il faut puiser des solutions et définir des conditions pratiques. "9

En d'autres termes, on ne saurait parler, à l'état actuel, d'une théorie pédagogique des grands groupes en Afrique, mais des pédagogies de grands groupes, adaptées chacune, à une situation spécifique différente. On peut avoir plusieurs situations d'enseignement/ apprentissage en grands groupes dans un même pays ou dans une même région. En d'autres termes chaque pays africain connaît des spécifiques de difficultés liées aux grands groupes; par conséquent considérer cette problématique sur le plan du continent africain tout entier, ou même de toute l'Afrique noire francophone participe d'une surgénéralisation. Cependant que ce soit au niveau national ou régional, l'un des défis les plus urgents à relever consiste à harmoniser les pratiques de classe en usage dans ces différentes situations, et partant, à faire une de synthèse des résultats des expériences pédagogiques isolées, ce qui servirait de base à une esquisse de théorisation de la pédagogie des grands groupes.

Publié par la CONFEMEN en 1991 le Répertoire méthodologique sur les techniques d'organisation et d'enseignement dans les classes à effectifs pléthoriques est un point de départ appréciable pour une réflexion approfondie sur la question. Ce répertoire, dont la rédaction avait été commandée par les Ministres de l'éducation des pays francophones lors de la $38^{\mathrm{e}}$ session de la CONFEMEN en 1989 comporte une quinzaine de fiches techniques proposant chacune des possibilités variées d'organisation de la classe et d'enseignement en grands groupes. D'après les auteurs, ces fiches sont présentées "dans toute leur généralité pour pouvoir être essayées dans les conditions matérielles des contextes institutionnels, des orientations pédagogiques extrêmement divers." ${ }^{10}$ Ces fiches, toutes issues d'expériences de terrain, ont été expérimentées pendant plusieurs années dans certains pays, dont le Cameroun, avec très peu de succès sur le plan pédagogiques. D'autres pistes sont actuellement expérimentées dans divers pays africains. En marge des travaux de terrain, la formation continuée des enseignants aux techniques d'organisation et de gestion des grands groupes a été amorcée il y a une quinzaine d'années sous les auspices de la CONFEMEN

16 Afin de transformer ce qui, pour la plupart des pays africains, semble être une fatalité en une situation humainement gérable, des efforts de différents types ont été entrepris et se poursuivent dans divers pays, même si au Cameroun l'on semble s'accommoder de la situation. Il est probable que dans les décennies à venir, des solutions durables seront trouvées par ces pays, soit en accélérant les travaux de recherches en pédagogie des grands groupes, soit en réformant les systèmes scolaires actuels, pour les adapter aux réalités locales, et plus particulièrement aux problèmes de la croissance démographique. En attendant, des stratégies de remédiation à court et moyen termes sont mises en place, pour une amélioration de l'enseignement du français dans les pays du Sud, notamment avec l'aide de l'Agence de Coopération Culturelle et Techniques et des bâilleurs de fonds internationaux. Il ressort des conclusions des travaux des différents séminaires et réunions relatifs à la pédagogie des grands groupes ${ }^{11}$ que la recherche de solutions durables suppose le recensement préalable des causes de l'accroissement de la demande de scolarisation et des conséquences découlant de cette situation, la formulation des propositions pédagogiques concrètes, etc. Au Cameroun, la réflexion, encore embryonnaire, ne semble pas constituer une priorité pour le pouvoir; pourtant le 
phénomène des grands groupes prend de l'ampleur, aussi bien au niveau primaire que secondaire, voire au niveau universitaire. L'enseignement supérieur, il connaît même une situation plus tragique, en dépit de l'éclatement en 1993 de l'Université de Yaoundé en six universités d'Etat, auxquelles il faut ajouter l'Université Catholique d'Afrique Centrale. Le bilan des vingt ans de réflexion sur la pédagogie des grands groupes en Afrique noire demeure dans l'ensemble assez maigre; il se limite, pour l'essentiel, à quelques expériences pilotes menées au niveau primaire, et dans de très rares cas, au niveau secondaire. Pourtant une réflexion plus approfondie sur la gestion des grands groupes aux niveaux secondaire et supérieur s'impose, si l'on veut améliorer la qualité de l'enseignement du français en particulier, et les conditions de fonctionnement de l'école en général.

\section{Recherche d'unicité en didactique du français.}

Comme nous l'avons souligné supra, la situation actuelle étant appelée à durer pour longtemps encore, il est souhaitable voire recommandable qu'une politique d'harmonisation des pratiques pédagogiques dans les grands groupes soit mise en place au Cameroun de toute urgence, même si à long terme, on ne saurait cependant se résigner à une telle hypothèse, qui est non seulement trop coûteuse, mais aussi peu rentable. Néanmoins face à la croissance exponentielle continue de la population scolarisable et la diminution drastique des moyens financiers, situation rendue encore plus catastrophique avec l'avènement de la crise économique qui sévit au Cameroun depuis une vingtaine d'années, espérer un changement positif rapide de la situation relève d'une vision chimérique. Cependant la nécessité et l'urgence de la refondation de l'école et d'une redéfinition de sa place dans la diffusion du français imposent de nouvelles pistes de réflexion.

Parmi celles-ci, celles relatives aux approches didactiques méritent une attention particulière. En didactique des langues en général, il existe un certain nombre d'invariants méthodologiques que les enseignants en situation de grands groupes ont tout intérêt à maitriser. Si l'on admet qu'en situation de grands groupes, l'enseignant est surtout confronté au problème de gestion pratique de la classe : discipline, notation, correction et appréciation des devoirs, animation du groupe, dynamique interactionnelle ; outre ces problèmes ayant partie liée avec le nombre élevé d'élèves, il n'y a pas de divergences fondamentales entre la didactique du français dans les petits groupes et dans les grands. Il n'y a pas de cloisons étanches ou de barrières infranchissables entre les deux situations d'enseignement. Au-delà des spécificités qui ont partie liée aux contextes politiques, sociolinguistiques et économiques, la didactique des langues en général présente une unicité et de nombreux invariants. Certes les pratiques méthodologiques divergent, mais dans le fond, les pratiques didactiques des langues maternelles, secondes ou étrangères présentent de nombreux points de convergence. Une véritable synergie devrait s'établir entre les enseignements dans les petits groupes pratiqués dans les pays du Nord, et les situations de pédagogie des grands groupes qui s'imposent fatalement dans les pays du Sud. En d'autres termes, l'une des solutions possibles au problème des classes à effectifs élevés pourrait être la mise en place des réseaux d'échanges pédagogiques entre les pays du Nord et ceux du Sud d'une part, et entre les pays du Sud d'autre part. Les enseignantsdidacticiens du Sud et ceux du Nord ont donc intérêt à travailler ensemble à la mise en place des banques de données méthodologiques, car en s'enfermant chacun dans une 
logique méthodologique isolationniste, ils ne pourront pas bénéficier des expériences réciproques menées dans les divers contextes. De même qu'il n'existe pas de méthodologie spécifique du français langue seconde, coupée de la didactique des langues en général, on ne saurait imaginer en Afrique une didactique spécifique aux grands groupes, fondamentalement différente de celle des petits groupes. En effet la plupart des manuels de français et des outils méthodologiques utilisés en pédagogie de grands groupes en Afrique sont conçus et réalisés dans les pays du Nord, où la tendance est plutôt à la pédagogie des petits groupes. Mais la diversité des situations sociolinguistiques impose l'adaptation de ces outils aux réalités locales, culturelles et sociales.

L'objectif partagé de l'enseignement du français comme langue maternelle en France, ou comme langue seconde dans les pays du Sud, est de favoriser son usage comme langue de scolarisation ou d'acquisition des savoirs. Il est regrettable cependant de constater que dans la plupart des pays du Sud, les systèmes scolaires fonctionnent aujourd'hui encore sur le modèle des programmes, objectifs et finalités identiques à ceux en usage au XIX siècle dans les pays du Nord, alors que les exigences culturelles et sociolinguistiques de la didactique du français dans les grands groupes appellent à des changements radicaux, et à des réformes pédagogiques fondamentales. La rigidité des horaires et des programmes est en fait incompatible avec la nécessité d'adapter l'organisation des enseignements aux exigences des grands groupes, où les inter-actions impliquent une large variation et une grande diversification des modes d'intervention. Car les stratégies didactiques mises en place par l'enseignant en classe de langue sont la condition sine qua non de la réussite de toute action pédagogique.

En didactique du français en particulier, et des langues en général, la loi de la variété requise, c'est-à-dire la pluralité des modes d'intervention pédagogique s'impose plus qu'ailleurs. Car les exigences didactiques, en situation des grands groupes ne sauraient s'accommoder de la rigidité des emplois du temps traditionnels, de l'utilisation du temps dans le groupe-classe, et des rôles classiques immuables de l'enseignant qui enseigne et de l'apprenant qui apprend. La variété des profils des apprenants et des situations d'apprentissage impose une différenciation des objectifs, des stratégies et des modalités organisationnelles du travail des élèves. Ceci implique donc que l'enseignant a une assez large maitrise des théories qui sous-tendent les différentes approches méthodologiques, qu'il est capable d'en faire la synthèse, afin de tirer le meilleur de chacune d'elles, d'où l'urgence de l'institution de la didactique comparée comme discipline dans les écoles normales et comme un nouveau champ de recherches.

\subsection{Pédagogie des grands groupes et pédagogie différenciée}

21 Par essence, le grand groupe est un groupe hétérogène : ses membres viennent d'horizons divers, avec des héritages linguistiques et culturels différents et des habitudes d'apprentissage diverses. Cette hétérogénéité impose en pédagogie des grands groupes une distribution des tâches et des rôles, différente de celles qu'on trouve dans les situations classiques en didactique des langues. Cette hétérogénéité impose à l'enseignant l'adaptation de ses stratégies de communication du savoir à la nature hétéroclite du groupe-classe, en d'autres termes, en optant par exemple pour une pédagogie différenciée, quand cela est possible. Une pédagogie est dite différenciée, lorsqu'elle permet d'individualiser les apprentissages, en reconnaissant à chaque élève une personnalité propre, et des attitudes personnelles face à l'apprentissage. 

cognitives linguistiques et psychologiques de l'élève, et s'oppose ainsi à l'idéologie de l'égalité de tous les élèves devant le savoir, et au mythe identitaire de l'uniformité que véhicule cette idéologie, qui, paradoxalement, semble être à la base de la réflexion sur l'école en Afrique noire en général et au Cameroun en particulier. Son objectif principal est donc la réduction des échecs scolaires, en prenant compte de l'hétérogénéité du groupe-classe, et en adaptant l'enseignement et l'évaluation aux conditions spécifiques de chaque élève ou de chaque groupe d'élèves, c'est-à-dire en mettant en place des processus qui permettent de débloquer chez eux le désir d'apprendre, de trouver chacun sa propre voie d'accès au savoir, en un mot, d'améliorer les relations entre enseignés et enseignants. La différenciation de l'action pédagogique est le résultat de la reconnaissance de la responsabilité fondamentale du dysfonctionnement du système scolaire dans l'échec des élèves. Elle porte sur les méthodes d'apprentissage, les contenus des programmes et la structuration de la classe. Nous pensons avec Halina Przesmycki que différencier la pédagogie nécessite d'abord une différenciation des structures. Selon elle, "une pédagogie différenciée authentique est celle qui est fondée sur la différenciation des processus d'apprentissage des élèves et qui passe, pour atteindre ce but, par l'organisation [...] diversifiée et variée des processus d'enseignement." ${ }^{12}$ Ainsi, la pédagogie différenciée peut être l'une des solutions possibles au problème des classes à effectifs élevés, dans la mesure où elle permet une distribution des rôles, et une diversité des stratégies au sein du groupeclasse, offrant ainsi à l'enseignant l'opportunité de rompre avec l'immobilisme traditionnel des structures organisationnelles classiques.

La pédagogie différenciée apparaît donc comme l'une des voies capables de réduire la mortalité scolaire, en améliorant la qualité de l'enseignement du français au Cameroun. En prenant en compte l'hétérogénéité des élèves et donc, en s'intéressant particulièrement à ceux en difficulté scolaire l'enseignant cesse d'être un dispensateur des savoirs, et devient l'animateur du groupe-classe. Il convient de se demander si l'école au Cameroun offre les possibilités structurelles et matérielles d'une démarche pédagogique axée sur l'enseignement différencié. En d'autres termes, permet-elle de concilier un système d'enseignement/apprentissage rigide et linéaire, basé sur des programmes et des manuels dont les contenus doivent être suivis à la lettre, et s'adressant à la masse d'une part, et l'idéal d'un enseignement différencié, qui prend en compte les problèmes spécifiques de chaque élève d'autre part; ce qui suppose une plus grande marge de manœuvre vis-à-vis des instructions officielles, des programmes et des horaires?

Sur ce plan, l'enseignant camerounais est doublement limité dans ses initiatives visant à prendre en compte les spécificités des élèves en difficulté scolaire. Il est tenu de couvrir les programmes officiels, découpés en séquences rigides imposant des délais à respecter, ponctuées d'évaluations dont l'organisation dépend généralement des instances externes à l'enseignant. Par conséquent, il est obligé de progresser, non pas au rythme des élèves, mais à celui des textes et règlements qui fixent les échéances à respecter. Il avance à une cadence imposée par les programmes officiels, dont le caractère atomisé, et sans lien intrinsèque apparent, l'oblige quelquefois d'accélérer son rythme de progression, de sauter des sections pourtant essentielles, voire de souvent passer sans transition d'une unité didactique à une autre. Ces programmes officiels, conçus par le Ministère de l'éducation nationale, et destinés à un public abstrait, ne prennent pas en compte les besoins individuels des apprenants, ni les spécificités des conditions d'enseignement/ apprentissage qui varient souvent d'une région à une autre, à l'intérieur du pays. 
exemple, au Cameroun les situations d'enseignement du français ne sont pas identiques dans les villes et en zones rurales, ni dans les quartiers résidentiels et les bidonvilles. Cependant, en dépit des inégalités linguistiques, culturelles et économiques criantes entre les deux milieux, les programmes, horaires, objectifs et contenus fixés par le Ministère de l'éducation sont identiques pour tous les jeunes Camerounais. Pourtant théoriquement, on ne devrait pas enseigner le français aux enfants issus des milieux sociaux culturellement avantagés, donc qui en ont déjà une certaine maîtrise, avec les mêmes outils méthodologiques que ceux employés avec les enfants originaires des milieux sociaux défavorisés, et pour qui il a généralement le statut de langue étrangère stricto sensu. En effet le caractère démocratique de l'école, et surtout la prétention démagogique des politiques de donner à tous les élèves les mêmes conditions de réussite constituent l'un des obstacles majeurs à la pédagogie différenciée. Il faut reconnaitre que cette prétendue égalité des chances pour tous, prônée par l'école, est une vraie utopie car, pour reprendre Pierre Bourdieu,

"pour que soient favorisés les plus favorisés et défavorisés les plus défavorisés, il faut et il suffit que l'école ignore, dans le contenu de l'enseignement transmis, dans les méthodes et les techniques de transmission et dans les critères de jugement, les inégalités culturelles entre les enfants des différentes classes sociales : autrement dit, en traitant tous les enseignés, aussi inégaux soient-ils en fait comme égaux en droits et en devoirs, le système scolaire est conduit à donner en fait sa sanction aux inégalités initiales devant la culture. "13

Quelles que soient les difficultés que pose sa mise en place, la pédagogie différenciée apparait pourtant comme une solution fortement envisageable au Cameroun, si l'on veut réduire le taux de mortalité scolaire, et améliorer la qualité de l'enseignement du français, aussi bien comme unique vecteur d'acquisition des autres savoirs, que comme principal outil de communication. Dans un contexte où l'enseignement traditionnel, qui s'adresse aux masses a montré toutes ses limites, il urge substituer à l'approche pédagogique classique, une approche nouvelle, qui prend en compte les diversités socioculturelles et linguistiques des élèves et, les disparités liées à l'hétérogénéité des classes, et à la différence de niveau socio-économique et professionnel entre leurs familles. Cette démarche pédagogique cherche à réduire ainsi les inégalités de traitement entre les élèves. Comme le souligne P. Burns, "il n'y a pas deux apprenants qui progressent à la même vitesse. Il n'y a pas deux apprenants qui soient prêts à apprendre en même temps. [...] Il sera donc opérationnel de mieux connaitre les élèves à la fois dans l'hétérogénéité de leur cadre de vie non scolaire et scolaire, puis dans leurs processus d'apprentissage." 14

\subsection{L'enseignement mutuel}

Dans le grand groupe, la discipline est l'un des problèmes fondamentaux à résoudre : plus sa taille est grande, plus il est difficilement gérable. Parmi les expériences et initiatives isolées entreprises par quelques enseignants camerounais de façon informelle pour faire face aux grands groupes, il convient de citer l'enseignement mutuel, qui consiste à responsabiliser certains élèves, en leur confiant une part des tâches incombant normalement au maître. Ainsi, dans une classe, on peut avoir, en fonction de leurs compétences avérées, et de leurs motivations, des élèves responsables des leçons de grammaire, d'orthographe ou de calcul; leur rôle étant d'aider les élèves les plus faibles à faire leurs devoirs et à réviser leurs leçons après le cours. D'autres tâches élémentaires peuvent également leur être confiées : lecteurs de textes, effaceurs de tableau, ou même 
d'écrire les résumés et les exercices au tableau, etc. La responsabilisation des élèves, en leur confiant, de temps de temps, des tâches secondaires peut constituer une véritable source de motivation et de stimulation pour la classe, dans la mesure où chaque élève voudrait se voir confier ces tâches. Dans le système à double flux, on peut faire intervenir des élèves des classes supérieures dans les petites classes, éventuellement réparties en sous-groupes; leur rôle consistant à aider les jeunes élèves à assimiler leurs leçons et à répéter les leçons particulièrement difficiles après la classe. Certains parents d'élèves interrogés ${ }^{15}$ pensent cependant qu'il est anormal que l'enseignant se décharge ainsi, même partiellement, sur ses élèves, qui n'ont déjà pas suffisamment de temps à consacrer à leurs études, et qui surtout n'ont pas une formation adéquate. Pour eux le temps consacré par ces élèves aux tâches secondaires d'encadrement d'élèves plus faibles peut servir à d'autres apprentissages. Selon eux, demander à un élève, même plus intelligent, d'enseigner à d'autres enfants, c'est comme si on demandait à un patient moins souffrant, de soigner d'autres patients, qui justement parce qu'ils sont plus souffrants, ont le plus besoin du médecin. Car pour eux, chaque élève en situation de difficultés scolaires est un cas unique, qui requiert une attention particulière de la part de l'enseignant, c'est-à-dire qui a besoin d'un enseignement personnalisé.

Ainsi, pour ses détracteurs, l'enseignement mutuel ne saurait constituer une solution au problème des grands groupes au Cameroun. Selon eux l'enseignement mutuel est non professionnel, et par conséquent, il est anormal que le sort des élèves plus jeunes, et donc psychologiquement plus fragiles soit confié à des autres élèves, même plus avancés. Il est donc pour eux inadmissible de réexpérimenter des pratiques qui ont montré toutes leurs limites ailleurs. En outre, certaines personnes interrogées pensent qu'en confiant l'instruction des élèves plus jeunes à des pseudo enseignants inexpérimentés, et sans formation, ils courent le risque d'être exposés aux fautes et erreurs qu'il est plus tard difficile d'éradiquer.

Pourtant, les défenseurs de l'enseignement mutuel pensent au contraire qu'on peut bien s'appuyer sur l'expérience menée dans d'autres pays pour tenter de résoudre le problème des grands groupes au Cameroun, car c'est sur les ruines du passé qu'on bâtit l'avenir. Un argument de poids en faveur de l'enseignement mutuel permet de soutenir la thèse des défenseurs de l'enseignement mutuel : l'expérience montre que c'est grâce à l'aide des enfants plus âgés que les jeunes enfants apprennent toutes sortes de jeux; d'autre part, dans l'Afrique traditionnelle, l'initiation des plus jeunes était confiée aux plus jeunes. En outre certains défenseurs justifient leur prise de position en faveur de cette forme d'enseignement par le fait qu'ils ont souvent vécu eux-mêmes cette expérience, qu' A. Sadji rapporte dans L'Education africaine:

"les élèves étaient répartis en sections, confiées chacune à un moniteur, et ces moniteurs n'étaient autres que les élèves plus avancés. Chaque matin, avant que l'école ne s'ouvrît à l'ensemble, l'instituteur, pendant une heure ou deux, réunissait les moniteurs, complétait leur instruction générale, leur faisait répéter les leçons qu'ils allaient avoir à donner à leurs camarades et surtout (il) leur inculquait des procédés d'enseignement et de discipline. "16

Nous avons nous-même été formé au niveau primaire dans ce système: au cours moyen deuxième année, bien qu'étant l'un des plus jeunes de la classe, nous avions secondé le maître tout au long de l'année; il nous est même arrivé de le remplacer toute une journée entière. Le souvenir de cette expérience nous permet que l'enseignement mutuel est tout aussi bénéfique pour l'élève à qui on confie la charge de la classe, qu'à ses camarades; nous pensons que les jeunes peuvent apprendre mieux lorsqu'ils ont entre eux. 
Cependant, la logique voudrait que les enfants en difficultés, scolaires soient confiés à des enseignants plus expérimentés, car pour reprendre P. Perrenoud, "c'est lorsque la réalité résiste qu'il faut des compétences professionnelles spécifiques, lorsqu'on se trouve en face d'un enfant peu motivé, peu soutenu par le milieu familial, ou rencontrant des difficultés"17.

31 Pourtant l'expérience montre qu'au Cameroun les basses classes, qui sont réputées difficiles, sont confiées généralement aux jeunes enseignants souvent très peu expérimentés. Une telle pratique très courantes au niveau primaire contribue largement à augmenter les échecs et redoublements scolaires. Car c'est dans ces classes qu'on rencontre non seulement les effectifs les plus élevés, mais aussi le plus grand nombre de cas de déviances, de marginalisation, d'inadaptation scolaires. Par conséquent, nous pensons qu'il est souhaitable de réduire au minimum les effectifs dans ces petites classes, et les confier à des enseignants chevronnés. En réduisant la taille des petites classes au minimum l'enseignant permet aux élèves l'acquisition des éléments de base et les fondements de la langue, à travers une meilleure organisation des activités conversationnelles. Il est évident qu'une gestion efficiente des grands groupes est fonction de l'aptitude de l'enseignant à les fractionner en autant de petits groupes, en fonction des types d'activités. En définitive, sa capacité à bien gérer les situations de grands groupes dépend de son aptitude à transformer l'obstacle que constitue la loi du grand nombre en avantage pédagogique.

Il se dégage de la micro-enquête citée supra que les initiatives en vue d'améliorer la qualité de l'enseignement sont de plus en plus sollicitées par les parents d'élèves. A travers des circuits de formation parallèles, qui ont pour vocation de permettre aux élèves d'acquérir des compléments de connaissances ou de réviser leurs leçons : cours du soir, cours complémentaires organisés par les enseignants, cours de vacances, recours aux répétiteurs, ils essaient ainsi de pallier, tant bien que mal, aux lacunes contre lesquelles l'école classique ne peut faire face. Le recours aux "répétiteurs" et autres circuits parallèles de formation est une pratique courante au Cameroun. Individuellement ou en groupes, des enseignants et étudiants organisent des cours de répétition à l'intention des élèves du primaire et du secondaire, surtout ceux qui sont en classes d'examen. Depuis quelques années, face à l'échec du système scolaire camerounais, et à l'impuissance du gouvernement à arrêter la dégradation de la qualité de l'enseignement, les parents recrutent des répétiteurs, ou s'organisent en groupes, au niveau des quartiers, pour embaucher des enseignants, qui s'occupent de leurs enfants après les classes. Cette pratique permet aux élèves de réviser leurs leçons, sous la supervision d'enseignants qualifiés, qui peuvent ainsi arrondir leurs fins de mois, ou souvent avec l'aide des étudiants. En attendant qu'une enquête à large échelle soit menée au Cameroun pour déterminer l'ampleur réelle du phénomène et son impact sur les résultats scolaires, il n'est pas prouvé que ces pédagogies parallèles améliorent effectivement les performances des élèves. Mais il faut reconnaître que ces pratiques présentent cependant un danger certain : ces cours de répétition se transforment parfois en de véritables cours parallèles, qui ne respectent pas toujours les programmes officiels. Quelles qu'en soient les formes : cours de répétition, ou de vacances; ces enseignements parallèles ne sont qu'une forme accompagnement scolaire, c'est-à-dire un soin palliatif, qui confirme la faillite des approches traditionnelles. Par conséquent la solution durable au problème des grands groupes passe par une réduction drastique de la taille du groupe-classe. 


\subsection{Fondements pour une pédagogie des petits groupes}

33 En pédagogie des grands groupes, l'un des rôles de l'enseignant est la réorganisation dugroupe-classe en sous-groupes de taille acceptable, en fonction du type d'activités programmées. Or la notion de grand groupe qui est une notion relative dépend de plusieurs facteurs inter-reliés, on ne peut déterminer où finit le petit groupe et où commence le grand : un petit groupe peut aller de trois individus à plusieurs dizaines. Selon le contexte, une classe de vingt ou trente élèves peut être considérée comme une classe normale, ou comme une classe à effectifs pléthoriques. Un cours magistral à cent étudiants dans un amphithéâtre est normal; alors qu'un groupe de travaux dirigés de quinze individus peut être qualifié de pléthorique. Pour Didier Anzieu et Jean Yves Martin, un petit groupe est un groupe qui comprend un "nombre restreint de membres, tel que chacun puisse avoir une perception individualisée de chacun des autres, être perçu réciproquement par lui et que de nombreux échanges interindividuels puissent avoir lieu. ${ }^{18}{ }^{\mathrm{Q}} \mathrm{Quant}$ à G. C. Homans, il pense qu'"un petit groupe consiste en un nombre de personnes qui communiquent entre elles pendant une certaine période, et peu nombreuses pour que chacune puisse communiquer avec toutes les autres, non par personne interposée, mais face à face ${ }^{119}$. Il se dégage de ces définitions que le nombre de membres et la fonction communicative sont les deux critères définitoires du petit groupe. Si elles soulignent le caractère restreint du nombre de membres, elles n'en fixent pas le seuil indicatif. En revanche elles mettent l'accent sur l'interaction verbale entre les membres. En effet c'est sur le postulat qu'une société est faite d'individus qui se communiquent entre eux que se fonde l'interaction verbale en classe de langue, le lieu par excellence de communication. Or pour que la communication ne peut s'y dérouler dans des conditions normales que si les rôles sont clairement et si les élèves sont répartis en petits groupes. Cette répartition peut se faire suivant plusieurs modalités, la méthode la plus simple étant la séparation géographique des membres, suivant loi du hasard, ou à partir du découpage des listes des participants, ou encore par le tirage au sort. Mais elle peut se faire aussi en fonction de l'âge, du sexe; sur désignation par l'enseignant, (ou par les autres élèves) ; ou encore sur la base du volontariat (auto-formation des sous-groupes, sur la base des affinités, vote, inscription sur des listes, en fonction des thèmes). Les sous-groupes ainsi formés sont plus ou moins stables, et leur durée de vie varie en fonction de la nature des activités qui ont motivé leur création. En situation de pédagogie des grands groupes il ne suffit pas cependant, pour l'enseignant, d'organiser la classe en groupes, mais d'y jouer le rôle d'animateur et de véritable chef d'orchestre.

Si dans les pays développés la classe de langue ne dépasse pas une trentaine d'élèves, au Cameroun sa taille moyenne est d'une centaine voire de cent cinquante élèves ; or il est établi que l'apprentissage des langues en petits groupes présente plusieurs avantages : les élèves ont plus confiance en eux ; par conséquent, même les élèves les plus timides, qui souvent n'arrivent pas à s'exprimer dans une classe à effectif élevé, peuvent y prendre la parole, dans des situations de communication diverses. Cet apprentissage coopératif leur est bénéfique : il se développe entre eux l'esprit d'équipe. Contrairement à l'individualisme et l'esprit de compétition qui caractérisent le système scolaire traditionnel, l'apprentissage des langues en petits groupes peut être stimulant. Dans les petits groupes, l'élève développe des habiletés différentes de celles que privilégie le système scolaire classique : leadership, esprit d'équipe, aptitude à écouter les autres, aptitude à mener un groupe, habileté interactive, etc. Le petit groupe est aussi bénéfique 
pour l'enseignant, qui cesse d'être le simple distributeur du savoir, pour devenir le guide et l'organisateur de la communication et de l'interaction verbale. Les contacts enseignant-élèves sont plus directs et plus personnels que dans une classe traditionnelle. Il cesse d'être le dispensateur des savoirs, pour devenir un organisateur des connaissances. Quant à l'élève, il n'est plus un simple assimilateur des savoirs, mais un artisan de sa formation. L'école camerounaise, dans sa forme actuelle est le lieu où l'on apprend pour réciter; l'élève camerounais est réputé pour son obéissance et pour sa faculté à réciter. Or réciter n'est pas penser, l'école nouvelle a pour mission d'apprendre à penser et à s'exprimer.

\subsection{L'évaluation dans les grands groupes}

La variable du nombre constitue l'un des obstaclesmajeursauxquels est confronté l'enseignant camerounais. Si l'évaluation dans une classe d'une vingtaine d'élèves n'est déjà pas facile, sa tâche est plus dans une classe de cent-cinquante élèves. Rien de plus harassant en effet pour lui, que la correction des centaines de copies, dans les conditions psychologiques pas toujours enviables. Plus les effectifs sont nombreux, plus sa tâche devient difficile, et par conséquent la marge de subjectivité du correcteur est plus grande. Plus que dans les autres disciplines, l'évaluation et la correction des matières littéraires subit l'influence de la loi du grand nombre. Dans la pratique quotidienne de la classe, la notation des devoirs, qui constitue l'une des tâches de l'enseignant est d'autant plus difficile que le groupe est nombreux. Cependant une bonne organisation et surtout une programmation rigoureuse peuvent permettre à l'enseignant d'y faire face. Il convient sans doute d'évoquer une expérience menée il y a une quinzaine d'années lorsque nous étions confronté à des classes à effectifs pléthoriques. Notre démarche consistait soit à faire un choix rotatif des devoirs à corriger; soit à alterner systématiquement la correction de ceux-ci, c'est-à-dire à corriger les devoirs de chaque sous-groupe une semaine sur deux; soit encore à procéder à une évaluation fragmentaire. Notre expérience de praticien montre que cette forme d'évaluation, qui consiste à n'évaluer qu'un aspect particulier des savoirs ou des savoir-faire à la fois est très efficace. L'évaluation de l'introduction, de la conclusion, des transitions, de la rédaction d'un paragraphe descriptif ou descriptif, de la maîtrise de l'argumentation, permet à l'enseignant de focaliser l'attention sur un savoir ou un savoir-faire précis.

Une autre expérience de classe relative aux grands groupes est la correction des copies : pendant plusieurs années, lorsque nous enseignions au lycée nous avons pu contourner la difficulté liée à la situation de grands groupes, en impliquant les élèves dans la correction et à la notation de leurs propres devoirs. En fonction des types d'activités, nous régulièrement faisions recours aux élèves pour corriger eux-même leurs devoirs. Pour les exercices de grammaire et de dictée par exemple, chaque élève corrige lui-même son travail (auto-correction), ou celui d'une autre élève (hétéro-correction). Pour les évaluations mensuelles, et trimestrielles, pour rendre cette pratique plus objective, les copies à corriger étant anonymes; nous faisions généralement permuter les copies de nos différentes classes, de manière que les élèves d'une classe $\mathrm{A}$, corrigent les copies de la classe $B$ et vice-versa. Evidemment une telle pratique implique un travail minutieux dans la préparation et la rédaction d'une correction type et du barème de correction.

Toujours lorsque nous enseignions au lycée, nous avons expérimenté deux formes d'évaluation assez originales dans trois classes terminales comptant 120 élèves chacune. 
La première démarche consiste à répartir la classe en douze groupes de travail de dix élèves chacun; chaque groupe étant placé sous la responsabilité d'un élèvecoordonnateur. L'évaluation de la production écrite: dissertation française, contraction de texte, commentaire de texte est traitée en deux temps. Pendant la première phase qui dure heure, chaque élève fait individuellement le devoir, comme dans toute situation normale d'évaluation; son travail consistant à la recherche des idées et à l'élaboration du plan. Au terme de cette première heure, les élèves rejoignent leurs groupes respectifs; ensemble chaque groupe fait la synthèse des bonnes idées contenues dans les devoirs individuels, le coordonnateur, qui joue le rôle de secrétaire étant chargé de la prise des notes. La dernière phase est consacrée à la mise en forme et à la rédaction définitive du devoir, à remettre au professeur pour la notation. Après la correction chaque groupe reçoit ainsi une note collective.. Ainsi au lieu des cent-vingt copies, le professeur n'en corrige que douze. La correction en classe est pour chaque élève l'occasion de découvrir non seulement ses propres lacunes, mais aussi celles de son groupe, et d'améliorer son travail personnel qu'il conserve comme devoir-modèle. L'expérience a montré que cette activité donne lieu à une interaction, qui dépasse généralement le cadre du cours de français, et que certains groupes ainsi formés dans le cadre du cours de français deviennent de véritables groupes d'études. Cette approche présente à nos yeux un double avantage : non seulement elle réduit considérablement le nombre de copies à corriger; mais aussi elle contribue à la socialisation des élèves, et au renforcement de l'esprit d'équipe; le sort de tout le groupe.

L'autre type d'évaluation, qui n'est pas en soit une innovation, quoiqu'elle ne soit pas pratiquée au Cameroun a partie liée avec la nature particulièrement hétérogène. En effet l'une des principales caractéristiques des classes à effectifs pléthoriques au Cameroun est la très grande disparité de niveaux, d'âges, d'habitudes et de compétences d'apprentissage qui existe entre les élèves d'une classe. Face à ces groupes-classes, dont les membres présentent des comportements et habitudes d'apprentissage très différents, nous avions pensé qu'il était injuste d'administrer tous les élèves les mêmes évaluations, comme c'est la pratique dans le système scolaire camerounais. En nous fondant sur la loi de la variété acquiseformulée par B. Lussato, qui stipule qu'"un environnement très varié ne peut être contrôlé que par un système d'une variété au moins équivalente" ${ }^{120}$ nous avions introduit dans nos classes l'évaluation différenciée. Nous pensons en effet que compte tenu des disparités liées à l'âge, au statut social des élèves, et à leur niveau et de compétence, il est impossible à tous les élèves d'une classe d'avancer au même rythme. Par conséquent leur imposer la même vitesse d'acquisition des connaissances, ou les soumettre aux mêmes tests, c'est ignorer ces différences et particularités individuelles. D'où la nécessité d'organiser la classe en plusieurs sous-groupes, selon les compétences et les aptitudes des élèves. Or l'objectif premier de l'enseignement différencié décrit supra étant de permettre à chaque élève d'avancer à son propre rythme, il serait anormal de les soumettre tous aux mêmes évaluations. L'évaluation différenciée part du postulat qu'on ne doit comparer que ce qui est comparable. Par conséquent les progrès de chaque élève ne sont mesurables de façon objective qu'à l'intérieur de son sous-groupe. Car en évaluant avec les instruments identiques des groupes-classes hétérogènes, l'école contribue à renforcer l'inégalité sociale, qu'elle est censée combattre. Le principe de la différenciation dans l'évaluation suppose que les élèves ne sont soumis qu'à des tâches correspondant à leur niveau de compétence, et qu'ils sont évalués par rapport à euxmêmes, et non par rapport à l'ensemble du groupe-classe. 
39 L'évaluation différenciée, qui exige de chaque élève en fonction de ses moyens, fonctionne sur un principe simple : soit soumettre tous les élèves d'une classe à un même test comportant des difficultés croissantes soigneusement étudiées, de façon à permettre au groupe le plus faible de ne répondre uniquement qu'à la première série de questions, au groupe moyen de répondre, en plus de la première série, aux questions plus complexes, et ainsi de suite, soit les soumettre à des tests différents (par exemple des exercices à trous pour le groupe le plus faible, des questions à choix multiples pour le deuxième groupe, et des questions de production écrites, plus difficiles pour le troisième groupe), les exigences variant évidemment en fonction du niveau des groupes. En d'autres termes, l'élève est évalué par rapport à lui-même et à son sous-groupe, et non pas par rapport au groupe-classe, comme c'est le cas dans la pratique traditionnelle au Cameroun. Afin de créer l'émulation et la saine concurrence entre les membres d'un groupe, il avait été convenu au départ, avec l'accord de tous, que l'élève d'un groupe donné, qui totaliserait, au terme d'une série d'évaluations, un score égal ou supérieur à 15/20 à trois ou quatre évaluations successives, passerait dans le groupe immédiatement supérieur au sien. Inversement, l'élève qui totaliserait successivement cinq scores inférieurs au seuil-plancher fixé à 05/20 devait être rétrogradé au niveau immédiatement inférieur. Evidemment en fin d'année, les élèves sont soumis au même type de test; comme l'exige l'institution, mais sont admis en classe supérieure non pas par ordre de mérite mais e fonction des progrès réalisés dans leur sous-groupe tout au long de l'année scolaire. Le plus important, dans d'évaluation différenciée, c'est que les élèves sont évalués sur des bases plus équitables. Face aux protestations liées à l'incompréhension de notre démarche par certains collègues, cette expérience a été abandonnée au bout d'une année. Cependant, malgré ces réticences et résistances, nous pensons qu'aussi bien les pratiques d'évaluation actuelles, que le système scolaire dans son ensemble, appellent une refondation radicale.

\section{Pour une refondation de l'école et les implications métadidactiques}

Plusieurs travaux mettent en exergue le dysfonctionnement chronique de la machine éducative en Afrique noire et donc au Cameroun et soulignent le caractère aigu de la crise multidimensionnelle qui la secoue. Ainsi L.J. Calvet affirme que "tout le monde est unanime à souligner que ces systèmes sont dans un état de crise grave dont les causes peuvent être diverses et les effets néanmoins convergents : inadéquation de l'école aux besoins des divers publics concernés et par conséquent, des Etats (ce qui militerait en faveur de l'hypothèse d'une crise structurelle.)"21 Tout en reconnaissant que la francophonie se porte apparemment bien en Afrique, P. Peroncel-Hugo, constate néanmoins qu'en réalité il ne s'agit que d'une apparence, car selon lui, l'école y traverse une crise aiguë et "derrière cette roborative façade littéraire et populaire, presque tout le système d'enseignement primaire et secondaire sans l'aide duquel la francophonie ne sera bientôt plus, du moins en Afrique, qu'une vague nébuleuse créolophone a atteint un état de délabrement alarmant". ${ }^{22}$ Dans un rapport sur la situation du français en Afrique adressé au Premier ministre français, le député Jean-Pierre Fuchs écrit : "on observe l'essoufflement d'une scolarisation qui ne répond pas toujours aux attentes de promotion sociale des familles, [...] ce mauvais rendement de l'école est un signe de dysfonctionnement des systèmes scolaires" ${ }^{123}$. Ainsi, ces chercheurs sont tous unanimes : ces lacunes relevées seraient les signes avant-coureurs d'une mort imminente de l'école en 
Afrique noire. Pour les plus optimistes, elles sont l'expression de son incapacité à continuer à servir vecteur de diffusion du français en Afrique noire Paradoxalement, après avoir posé ces diagnostics généralement pessimistes, les chercheurs en éducation et autres spécialistes se perdent dans des propositions et suggestion irréalistes et peu applicables, ou susceptibles de ne pas donner des résultats satisfaisants. Parmi ces discours pessimistes sur la situation de l'école en Afrique noire, citons les travaux de Robert Chaudenson pour qui,

"l'échec des systèmes scolaires est patent, ils avancent inéluctablement vers une implosion finale que rend inévitable l'évolution démographique et sociale; (tenter de les réformer ou de les amender comme on songe, semble-t-il, revient à changer la canne blanche ou les lunettes noires d'un aveugle qui marche droit vers un précipice!)[...] il est impératif et urgent d'imaginer d'autres systèmes adaptés à des conditions et des perspectives différentes, appuyées sur des moyens nouveaux qui ne peuvent être que ceux de la communication de masse" ${ }^{24}$.

41 Après une trentaine d'années d'expérience de l'enseignement du français à tous les niveaux du système scolaire camerounais, y compris, pendant six ans, à des adultes en milieu extra-scolaire (au Programme de Formation Linguistique de la Présidence de la République), nous convenons avec Robert Chaudenson qu'il est inutile de continuer à imposer l'école, du moins dans sa forme actuelle, et qu'il faut trouver d'autres voies, si l'on veut qu'elle continue à remplir sa mission de formation de la jeunesse et de diffusion du français au Cameroun. Car depuis plusieurs années, l'école camerounaise est en panne. Il urge de trouver de nouvelles voies susceptibles de la sortir de son enlisement actuel.

Bien que le contexte économique ambiant ne soit pas de nature à envisager un changement en profondeur du système scolaire, il convient de reconnaître que seule une solution globale peut permettre d'en améliorer le fonctionnement. Son développement étant directement tributaire du développement socio-économique, sa faillite est sans aucun doute liée à celle de l'économie. On ne peut donc dissocier la situation désastreuse de l'école de la situation économique, politique et sociale catastrophique que connaissent les pays africains en général et le Cameroun en particulier. Robert Chaudenson constate que "cette école qui vise à apprendre tout le français à tout le monde n'apprend rien à personne." 25 Même si ce jugement peut paraître quelque peu sévère dans la mesure où il ne prend pas en compte les efforts fournis par certains pays, pour faire face à une croissance démographique galopante d'une part, et à la dégradation des économies d'autre part, il faut admettre que depuis l'indépendance, l'école hérité de la colonisation n'a pas effectué la mue nécessaire, les transformations indispensables pour son adaptation au contexte africain. Les causes du dysfonctionnement et de la faillite de l'école sont à la fois internes et externes. Ainsi, parmi les causes externes, pour reprendre Isabelle Deble, les responsables de la situation dans laquelle se trouve l'école en Afrique aujourd'hui, "ce sont surtout les Occidentaux, pédagogues en mal de terrain, faux éducateurs coopérants, décideurs, experts qui ont proposé des formes nouvelles et des expériences de toutes sortes pour résoudre ailleurs ce qui pose problème chez soi"26. Par ailleurs, les causes internes de ce dysfonctionnement sont surtout liées à la croissance démographique incontrôlée, aux crises économiques et à une gestion archaïque et à la gabegie des politiques africains figurent parmi les plus importantes

$43 \mathrm{Au}$ Cameroun les échecs et redoublements scolaires ont partie liée avec la mauvaise maîtrise du français, non seulement parce qu'il est la matière de base aux niveaux primaire et secondaire, mais aussi parce qu'il est la langue d'enseignement. En effet, la maîtrise des autres disciplines est conditionnée par celle du français, qui n'est pas 
simplement une matière comme les autres; mais la matière matricielle, sans laquelle l'acquisition des connaissances en général n'est possible. Il est donc évident qu'une mauvaise maîtrise du français est synonyme d'échec scolaire. Par conséquent toute recherche de stratégies de réduction du redoublement scolaire au Cameroun doit prendre en compte l'amélioration de la qualité de l'enseignement du français.

Parmi les mesures concrètes susceptibles de permettre d'améliorer la qualité de son l'enseignement, il y a une modification fondamentale de l'attitude de l'école face aux langues locales d'une part, et face aux normes endogènes d'autre part. Car en se considérant comme l'unique gardienne de la norme linguistique standard, l'institution scolaire, refuse de reconnaître l'existence des normes endogènes qu'elle condamne comme sous-normes. Elle ne prend pas en compte la langue circulante dans l'enseignement du français, ce qui contribue à développer chez les élèves l'insécurité linguistique. En effet, comme l'affirme Marie-Louise Moreau,

"la reconnaissance, par la communauté francophone, d'une et d'une seule norme linguistique, les conditions historiques de la diffusion du français sur le territoire africain, les modalités actuelles de son apprentissage sur ce continent, [... ]constituent un terreau propice à l'épanouissement, chez tout francophone africain, d'une robuste insécurité linguistique." 27

En effet les conditions d'appropriation du français en milieu scolaire au Cameroun sont fortement génératrices du sentiment d'insécurité linguistique. L'insécurité linguistique naît de la tension entre deux pôles normatifs : la norme standard a priori inaccessible, imposée par l'école, et la norme fonctionnelle, moins valorisée certes, mais la plus utilisée et la plus courante dans la pratique quotidienne de la langue. En effet l'une des principales causes de la mauvaise maîtrise du français au Cameroun est liée au fait que l'école recherche surtout la conformité à la norme standard, au lieu de privilégier l'efficacité de la communication. Nous pensons donc qu'il faut désacraliser le français et démystifier, voire démythifier l'école, qui pourrait ainsi prendre en compte les normes endogènes et non les stigmatiser comme étant une mauvaise maîtrise de la langue. Le problème fondamental n'est pas cependant la reconnaissance par l'école des normes endogènes, mais plutôt celui d'une exploitation pédagogique de celles-ci, à travers une réhabilitation des variétés locales. Au lieu de les condamner et de les censurer, elle pourrait faire comprendre aux élèves pourquoi certains mots, qui ne peuvent être employés en classe, sont admis dans la cour de récréation;ou en famille, en leur expliquant surtout où, quand et avec qui ils peuvent s'exprimer d'une façon ou d'une autre. En effet cette langue fonctionnelle, dépouillée des contraintes normatives que l'école impose n'est rien d'autre que la langue telle qu'elle se parle dans la communauté de l'enfant. Nous pensons avec Gabriel Manessy (1994 : 217) qu'"il suffirait que l'école cessât de faire obstacle à la diffusion de cette variété et que, sans pour autant renoncer à enseigner le français standard qui conserve ses propres domaines d'exercice, elle acceptât d'assumer une fonction de contrôle sur la mise à jour et sur l'évolution d'une norme endogène complémentaire et non concurrente du modèle académique"28 $\mathrm{Il}$ ne s'agit pas d'instituer un enseignement systématique des normes endogènes, mais plutôt de ne plus continuer à les rejeter catégoriquement ces normes endogènes qui devraient servir de point de départ pour une analyse des conditions favorables pour un meilleur enseignement du français au Cameroun.

46 Par ailleurs les langues locales devraient trouver leur place à l'école, carle fait de dénier à l'élève le droit de parler sa langue maternelle, comme le fait l'école au Cameroun, renforce chez lui le sentiment d'insécurité linguistique. Depuis l'époque coloniale, les 
méthodes d'enseignement du français en Afrique noire francophone participent de l'approche submersive, la langue maternelle de l'élève étant submergée par le français. Or il a été prouvé qu'une situation d'apprentissage où la langue de l'enfant, langue dans laquelle ont été vécues ses premières émotions et ses premières grandes expériences, celle avec laquelle est construite son identité, est malmenée ou dévalorisée, débouche inévitablement sur un bilinguisme soustractif. L'échec scolaire massif au Cameroun a partie aussi liée avec cette approche submersive, qui fait l'apologie de l'unilinguisme en français, seule langue de l'école. Pour qu'elle cesse d'être génératrice d'insécurité linguistique d'une part, et de développer chez les élèves un bilinguisme soustractif d'autre part une refondation de l'école et une réorientation de ses missions s'impose, par une introduction des langues nationales et une attitude plus souple à l'égard des variétés locales du français.

47 Enfin, l'une des causes des échecs scolaires est l'hétérogénéité des classes : dans une même classe coexistent généralement des enfants d'origines diverses, et de niveaux socioculturels différents. Si pour ceux des zones rurales l'apprentissage du français ne commence que vers cinq-six ans avec le début de la scolarisation; en zones urbaines on trouve souvent dans une même classe des élèves ayant eu une appropriation extra ou préscolaire du français, ceux issus des écoles maternelles, ayant eu une ou deux années d'apprentissage préscolaire du français, ceux provenant des familles où le français est une langue quasi-maternelle et ceux qui sont de vrais débutants. La classe de français est ainsi le lieu d'un véritable continuum que la didactique devrait prendre en compte, soit e créant des groupes à partir des tests diagnostics qui permettrait de constituer des groupes homogènes correspondant au niveau réel des élèves.

\section{Conclusion}

Ainsi, l'analyse des problèmes auxquels fait face le système scolaire camerounais nous a permis d'en souligner la complexité des interactions. Il se dégage de cette étude que les diverses parties prenantes devraient orienter leurs actions vers les recherches de solutions globales au problème des grands groupes. Il convient cependant de reconnaître que si dans l'ensemble les pays africains font face aux classes à effectifs pléthoriques, toute généralisation doit être nuancée. Car dans les zones rurales en particulier, ce problème est moins sévère que dans les grands centres urbains. En outre, du fait de la faible densité de la population dans certaines régions, il n'a pas la même envergure dans les tous les pays africains, voire dans les différentes régions d'un même pays. Par Ces larges variations régionales et nationales dissimulent une diversité importante entre différents pays et à l'intérieur de ceux-ci, et confirment que non seulement les généralisations en formes de lois sur la problématique des grands groupes sont plutôt rares et que le problème doit être analysé d'abord sur le plan local, si l'on veut améliorer l'enseignement du français au Cameroun. 


\section{BIBLIOGRAPHIE}

Amos Comenius, J. (1657), La grande didactique. Traité de l'Art universel d'enseigner tout à tous, cité par Claude Oliviéri, "L’enseignement mutuel", in Diagonales, $n^{\circ} 7$.

Anzieu, D. ; Martin, J. -Y. (1973), La dynamique des groupes restreints, Paris, PUF.

Bales, R. (1950), Interaction process analysis, Cambridge, Mass.

Bourdieu, P.,( 1966), "L’inégalité sociale devant l'école et devant la culture", in Revue française de sociologie, $\mathrm{n}^{\circ} 3$.

Candelier, M.,( 1996), Le Polyglotte, Paris, Supplément à Langues Modernes nº 1.

CONFEMEN(1991), Répertoire méthodologique sur les techniques d'organisation et d'enseignement dans les classes à effectifs pléthoriques, Dakar, CONFEMEN.

Homans, G. C., ( 1950), The human group, New York, Harcourt and Brace.

Perrenoud, P. (1994), La formation des enseignants entre théorie et pratique, Paris, L'Harmattan.

Porcher, L.,( 1992), "Omniprésence et diversité des auto-apprentissages", in Le Français dans le Monde. Recherches et applications, février-mars.

Przesmycki, H., (1991), Pédagogie différenciée, Paris, Hachette-Education.

\section{NOTES}

1. Titulaire d'un Doctorat Nouveau Régime en Sciences du langage, option linguistique et didactique des langues (Université Stendhal-Grenoble 3) et d'un Doctorat de troisième cycle en littérature française, il est également diplômé de l'Ecole Normale Supérieure. Pendant une vingtaine d'années, il a enseigné dans diverses institutions publiques au Cameroun, notamment au Centre Pilote du Programme de Formation Bilingue, Présidence de la République. Depuis 1996 il est enseignant de didactique du FLE (français langue étrangère), de linguistique française, et de littératures française et francophones à l'Université de Buéa, (Cameroun).Il enseigne également à la Faculté d'Education de la même université. Il est l'auteur de plusieurs articles en sciences du langage, en didactique du français langue étrangère et en littérature française.

2. Conférence des Ministres de l'éducation des pays ayant en commun le français

3. op. cit., p.13.

4. Ministère de l'Education nationale, La Réforme de l'enseignement primaire au Cameroun, Y aoundé, 26-29 mars 1973.

5. Henry Tourneux, Olivier Iyebi-Mandjeck, L'école dans une petite ville africaine, Maroua, Cameroun, Paris, Karthalla, 1994

6. Aline Cook, "Francophonie au Cameroun indépendant: discours politique et mise en pratique au Cameroun oriental", in A. Miguet, P. Corcoron, (éds.), Francophonie, Mythes, Masques et Réalités. Enjeux politiques et culturels, Paris, PubliSud, 1996, p167.

7. op. cit., p.77.

8. Jean Amos Comenius, La grande didactique. Traité de l'Art universel d'enseigner tout à tous, 1657, cité par Claude Oliviéri, "L'enseignement mutuel", in Diagonales, ${ }^{\circ} 7$, p.34.

9. André de Peretti, "Les lois des grands nombres", in Diagonales, nº 7, p.29. 
10. CONFEMEN, Répertoire méthodologique sur les techniques d'organisation et d'enseignement dans les classes à effectifs pléthoriques, Dakar, CONFEMEN, 1991, p.15.

11. Séminaire d'experts de la CONFEMEN sur Les problèmes organisationnels et pédagogiques liés à l'accroissement de la demande scolaire dans les Etats membres, Djibouti, 18-25. 12.85.

12. Halina Przesmycki, Pédagogie différenciée, Paris, Hachette-Education, 1991, p.17.

13. Pierre Bourdieu, "L'inégalité sociale devant l'école et devant la culture", in Revue française de sociologie, $\mathrm{n}^{\circ} 3,1966$, pp. 336.

14. P. Burns, "Methods for individualizing instruction", in Cahiers pédagogiques, $n^{\circ} 148-149, p .188$.

15. Toutes les références à l'opinion ou aux points de vue des parents d'élèves dans la présente étude sont tirées des résultats d'une micro-enquête menée il y quelques années auprès de quelques associations des parents d'élèves de la ville de Yaoundé, dans le cadre de notre thèse de doctorat en sciences du langage.

16. A. Sadji, L'Education africaine, Paris, Karthalla, p.68.

17. Philippe Perrenoud, La formation des enseignants entre théorie et pratique, Paris, L'Harmattan, 1994, p. 181.

18. Didier Anzieu, Jacques-Yves Martin, La dynamique des groupes restreints, Paris, P.U.F., 1973, p.23.

19. G. C., Homans, The human group, New York, Harcourt and Brace, 1950, p.25.

20. Bernard Lussato, cité par A. de Peretti, in A. Peretti et al, Recueil de processus et d'instruments d'évaluation formative, Paris, Institut Nationale de Recherches Pédagogiques, 1983, p.127.

21. Louis-Jean Calvet, "Le français dans l'espace francophone", in Didier de Robillard, Michel Beniamino (éds.), Le Français dans l'espace francophone, Paris, Champion, 1996, p.404.

22. P. Peroncel-Hugo, Le Monde, p.41. $\mathrm{n}^{\circ} 2012$.

23. Jean Pierre Fuchs, Quel avenir pour le français dans les pays d'Afrique noire et de l'Océan indien? Rapport au Premier ministre, Paris, 1991, p. 45.

24. Robert Chaudenson, "Plurilinguismes et développement en Afrique subsaharienne francophone: les problèmes de communication", in Cahiers des sciences humaines, vol. 27,303-4, 1991, p.311.

25. Daniel Baggioni, Robert Chaudenson, et al, Multilinguisme et développement dans l'espace francophone, Paris, Didier Erudition, 1989, p.37.

26. Isabelle Deble, "Différenciations ou uniformisations?", in Afrique contemporaine, $\mathrm{n}^{\circ} 172,1974$, p.15.

27. op. cit., p.103.

28. Gabriel Manessy, Le français en Afrique noire. Mythe, Stratégie, Pratiques. Paris, L'Harmattan, 1994, p.217.

\section{RÉSUMÉS}

Le problème de la pédagogie des grands groupes a été posé pour la première fois en mars 1984, lors de la réunion à Khartoum, de l'Association des Professeurs de Français en Afrique. Plus de deux décennies plus tard, la loi du grand nombre continue à s'imposer, comme une fatalité, dans les classes en Afrique noire, aussi bien aux niveaux primaire, secondaire, que supérieur. A partir de l'analyse cette problématique au Cameroun à l'aube du XXI siècle la présente étude suggère 
une refondation du système scolaire camerounais en particulier, afin de l'adapter au contexte socioéconomique et politique actuel.

The present research is an investigation study on the teaching/learning situation in most african country twenty years after the lunching in1984 in Khartoum Sudan, of the first reflexion on the teaching in larger groups in sub-saharian countries in general and in Cameroon. in particular. stemic approach of the teaching/learning of French as the first and second official language in Cameroon. The first part provides essential orientations to the teachig/learig situation in Cameroon. After a preliminary description the teaching/learning situation in Cameroon, since 1960 , the second part focuses on the particular situation of the description

\section{INDEX}

Mots-clés : classes à effectifs pléthoriques, didactique des langues, enseignement différencié, évaluation, évaluation différencié, grands groupes, groupes hétérogène, petits groupes

\section{AUTEUR}

DAVID NGAMASSU

Université de Buéa. Cameroun 